Penta-Prism Long Trace Profiler (PPLTP) for Measurement of Grazing Incidence Space Optics*

Shinan Qian

Sincrotrone Trieste

Padriciano 99, I-34012 Trieste, Italy

Haizhang Li

Continental Optical Corporation

New York, New York

Peter Z. Takacs

Brookhaven National Laboratory

Upton, New York 11973-5000

May, 1996

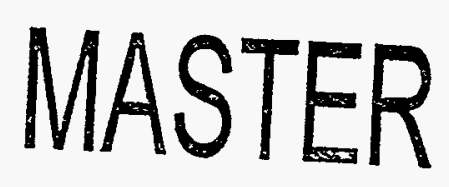

"This research was supported in part by the U. S. Department of Energy:

Contract No. DE-AC02-76CH00016. 


\title{
Penta-Prism Long Trace Profiler (PPLTP) for measurement of grazing incidence space optics
}

\author{
Shinan Qian \\ Sincrotrone Trieste, Padriciano 99, I-34012 Trieste, Italy \\ Haizhang $\mathrm{Li}$ \\ Continental Optical Corporation, New York, USA \\ Peter Z. Takacs \\ Brookhaven National Laboratory, New York, USA
}

\begin{abstract}
The Long Trace Profiler (LTP) is in use at a number of locations throughout the world for the measurement of the figure and mid-frequency roughness of $\mathrm{x}$-ray mirrors. The standard configuration requires that the surface tested lie in a horizontal plane as the optical head is scanned along a horizontal line. For applications where gravity-induced sag of the surface cannot be tolerated, such as in x-ray telescope mirror metrology, it is desirable to measure the mirror as it is mounted in a vertical configuration. By making simple modifications to the standard LTP system, we have demonstrated that it is possible to use the LTP principle to measure the surface of $x$-ray mirrors and mandrels mounted in the vertical orientation. The major change in the LTP system is the use of a penta prism on a vertical translation stage to direct the probe beam onto the surface and the addition of a precision rotation stage to hold the test object. A 3-D map of the surface topography of the complete cylindrical asphere can be generated quite easily with this technique. Measurements with a prototype system indicate a slope error accuracy of better than 1 microradian is possible, with a figure error repeatability of better than $50 \mathrm{~nm}$.
\end{abstract}

KEY WORDS: Optical testing, x-ray mirrors, metrology, surface figure, profilometry, LTP

\section{TEST REQUIREMENTS OF GRAZING INCIDENCE SPACE OPTICS}

Interest in $x$-ray astronomy has been growing throughout the world in recent years ${ }^{1}$. The number of space-borne $x$-ray telescope missions that have recently been launched or will be launched in the near future, are increasing. Some examples are ROSAT, SXO, SAX, AXAF, and XMM. The optical systems used to focus $\mathrm{X}$-rays in these telescopes are all based upon the use of grazing incidence optics in various configurations. Wolter Type I systems use a combination of paraboloidal and hyperboloidal surfaces; a Wolter-Schwarzchild Type I system consists of a small figure modification of a Wolter I system; the foil cone is an approximation of a Wolter I system, and the Kirkpatrick-Baez (K-B) system consists of two sets of orthogonal spheres or parabolic cylinders, produced by bending thin plates. All of these systems fall into the category of aspheric optics. The surface figures are generally conical in shape with very small sag deviations, on the order of microns, from the best-fit conical surface.

As the angular resolution of $x$-ray telescopes has improved in recent years from a few arc minutes to a few arc seconds, the requirements on the metrology for testing the surface figure and finish have become more stringent. In order to produce clear images and minimize scattered light at photon energies in the kilovolt range, surface roughness on the optics needs to be no greater than a few Angstroms. A number of commercial instruments are available for measuring the microroughness on optical surfaces with accuracy and repeatability below $1 \AA$. This aspect of surface metrology will not be addressed here. The more challenging problem is to measure the surface figure on these large cylindrical aspheres with the required accuracy.

In order to appreciate the test requirements of mirror surface figure, some specifications and test results of previously manufactured mirrors are quoted as follows:

a). The ROSAT high resolution $x$-ray telescope ${ }^{2}$ (Wolter type I, four nested mirrors, average grazing angle of about 2 degrees, maximum diameter of $835 \mathrm{~mm}$ ) produced by Carl Zeiss has an axial slope error of about 0.8 arc sec $\mathrm{rms}$, a differential out of roundness of about $0.8 \mathrm{arcsec}$ rms, and a telescope resolution of $20 \mathrm{arc} \mathrm{sec}$. 
b) A wide field camera/telescope, WFC, on the ROSAT satellite ${ }^{3}$ is a Wolter-Schwarzchild Type I grazing incluce, $\cdot$ e system with an angle of incidence of 7.5 degrees, a $576 \mathrm{~mm}$ diameter and a nest of 3 co-axial, confocal miiror: produced by NPL. The specifications require that the profile of any mirror should not deviate from the require 's form by more than $1 \mu \mathrm{m}$ over its axial length or depart from circularity by more than $12 \mu \mathrm{m}$ across its dianet $r$. What was achieved was: a $0.6 \mu \mathrm{m}(\mathrm{P}-\mathrm{V})$ deviation from the theoretical surface, a slope error of 5 arc sec and tek scope resolution of 2 are min.

c). A thin-foil, high-throughput $x$-ray telescope, XSPECT/SODART ${ }^{4}$, produced by the Danish Space Research Institutr, is an approximation of a Wolter I with an outer diameter of $600 \mathrm{~mm}$ and contains 154 nested mirrors. The resoluti' $n$ achieved is 2 arc min.

Surface figure requirements differ between high resolution telescopes and high throughput telescopes. For new high quality imaging $x$-ray optics, mirror figure errors must not exceed $0.5 \mu \mathrm{m}$ within the whole length, and slope errors must not exceed 0.5 arc sec. On the other hand, high throughput $x$-ray telescopes do not need surfaces with such high figure.accuracy: they need instead a low cost and versatile metrology instrument to test a large number of shells or foils at different manufacturing stages. Since these mirrors are conical aspheres, they are impossible to test with conventional interferometers. Consequently, high-quality $x$-ray telescope mirrors are manufactured only by a few large companies or advanced laboratories because most optical fabricators lack the precision measuring instruments and polishing machines to produce these surfaces. The penta prism LTP (PPLTP) offers the possibility for a metrology instrument that is optimized for measuring x-ray telescope mirrors and mandrels and that is accessible to all.

Some machines currently used in the testing of grazing incidence aspheres, and their characteristics, are as follows:

a) M400 co-ordinate measuring machine 5

Contact method - a mechanical probe contacts the surface under test. An interferometer measures the distance

between the probe and a reference surface.

$\begin{array}{ll}\text { Developed by } & \text { Carl Zeiss } \\ \text { Slope accuracy } & 0.1-0.2 \text { arc sec } \\ \text { Test range } & 3-D 400 \times 600 \times 200 \mathrm{~mm} \\ \text { Slope range } & \text { relatively large } \\ \text { Cost } & \text { high }\end{array}$

b) Laser Autocollimation Profilometer(LAP) 6

Non-contact method - a single collimated laser beam is scanned across a surface by a penta-prism. The

reflected beam is detected by a position sensitive detector which measures the slope of the surface .
Developed by
NPL
Repeatability
Teșt range
Slope range
Cost
$1 \mathrm{~nm}(80 \mathrm{~mm} \mathrm{scan}) / 10 \mathrm{mins}$
$200 \times 200 \mathrm{~mm}$ (plane)
moderate
low

c) Long-Trace-Profiler (LTP)

Non-contact pencil-beam interferometer

$\begin{array}{ll}\text { l)eveloped by } & \text { BNL/ALS/ELETTRA } \\ \text { lepeatability } & 0.04 \text { arc sec rms/ } 60 \text { mins } \\ \text { Slope accuracy } & 0.1-0.2 \text { arc sec rms(400 mm scan) } \\ \text { Test range } & 1-D \text { 1000-2000 mm ; 3-D extendible } \\ \text { Slope range } & \text { small, 10 mrad } \\ \text { Cost } & \text { low }\end{array}$

d) Other meillods.

An (t) the method for the assessment of the quality of thin foil mirrors was used by Kunieda and Serlemitsos 7 and the Darish space Research Institute 4 . It involves scanning a laser beam over the surface and looking for departures from thr prricticted laser beam position measured by a CCD camera produced by surface "orange peel". The system 


\section{DISCLAIMER}

Portions of this document may be illegible in electronic image products. Images are produced from the best available original document. 
has a resolution of $2.4 \mathrm{arc} \mathrm{sec}$. For the test of radius variation, a point source is put at the center of the cylinder in order to monitor the image position. In this way it is possible to know the surface figure and slope error pardy.

\section{PENTA-PRISM LONG TRACE PROFILER (PPLTP)}

The LTP is a precision surface slope angle measurement instrument. The measurement technique is based on the pencil-beam interferometer developed by von Bieren ${ }^{8}$ which was adapted by Takacs, Qian, et al. at BNL for measuring synchrotron optics. ${ }^{9-11}$ The LTP scans an unfocused laser beam across a surface without the need for auxiliary reference surfaces or null lenses. As such, it is a very versatile measurement system and can easily adapt to the measurement of most cylindrical spheres or aspheres, either concave or convex. The commercial version, LTP II, incorporates improvements suggested by Irick and McKinney at the ALS to use an internally-generated reference beam to correct for errors in the optical head during the scan motion. 12-15. Both the LTP and the LTP II utilize an air bearing translation stage to scan the optical head over the surface under test. To minimize dynamic errors and improve instrument performance, Qian, et al. ${ }^{16-17}$ moved the optical head off the translation stage onto a stationary mount and replaced it with a simple penta prism, as in the original concept of von Bieren ${ }^{8}$ This is the so-called penta-prism LTP (PPLTP) configuration. The LTPs at ELETTRA and at Continental Optical Corp. are now using the PPLTP configuration for routine operation.

When the LTP II is changed to the PPLTP, a number of new applications for the system become evident: it is now possible to test a mirror in situ under actual operating conditions, even in ultra-high vacuum, ${ }^{18}$ and it is now possible to scan the small penta-prism into the internal surface of conical cylinders, such as those used to make x-ray telescope optics.

The PPLTP schematic is shown in Fig.1. A polarized He-Ne laser and polarization-preserving optical fiber (POF) are used to deliver the laser pencil beam to the optical head. Collimated light from the fiber is sent into a non polarizing beamsplitter(BS) and then into two right-angle prisms (RP1 and RP2), which produce a pair of collinear beams with an adjustable separation distance. This beam splitting arrangement produces two exactly parallel beams with zero optical path difference. The beam pair is then split further into two pairs of beams, a test beam pair and a reference pair, by a polarizing beamsplitter (PBS). The set polarized parallel to the y axis is reflected by the PBS, while the other set, polarized parallel to the $x$ axis, passes through the PBS. Each set is reflected by either the mirror under test or the reference, then they retrace their paths through the system back to the PBS. The quarter-wave plates (QWP1,QWP2) swithch the polarization direction of each beam pair, and they are both sent through the PBS into the Fourier-transform lens system (FT). The detector array (DET) is placed at the focus of the lens so that the position of the focal spot is linearly related to the angle of the incident beam. A linear polarizer assembly $(P)$ is used to adjust the total intensity in the beams. A half-wave plate (HWP) between the non polarizing BS and the PBS is used to adjust the relative intensity between the test and reference beams. The test beam can be scanned by using a penta-prism (PT) mounted on a linear translation device. As the surface is scanned, the angle of the reflected test beam depends on the local slope of the surface, which is measured by analyzing the interference pattern in the image formed on the detector array. This is the slope profile which can be converted into height data by integration.

The PPLTP profits from the fact that the deflection angle at the penta-prism remains unaltered even though the prism may tilt slightly during scanning. This eliminates the need to correct the data for pitch angle errors in the motion of the optical head along the slide, as in the standard LTP II configuration. With these improvements, the remaining systematic errors in the PPLTP are reduced to below $0.5 \mu \mathrm{rad} \mathrm{ms}$ (or 0.1 arc sec) for plane or almost plane mirrors and to below $1 \mu \mathrm{rad} \mathrm{rms}$ (or $0.2 \mathrm{arc} \mathrm{sec}$ ) for mirrors with smaller axial curvature that deflect the test beam over the entire system angular acceptance range of $-4 \mathrm{mrad}$ to $+4 \mathrm{mrad}$. This accuracy is sufficient to meet the requirements of most grazing incidence space optics today and into the near future.

\section{TELESCOPE MIRROR TEST WITH A VSLTP PROTOTYPE}

The standard LTP II measures surfaces in the horizontal plane. The ideal configuration for measuring $x$-ray telescope optics is to measure the object while it is oriented with its symmetry axis in the vertical direction. This minimizes the effects of gravity-induced distortion on the surface figure, especially on thin shells or foil surfaces. Utilizing the PPLTP principle, a prototype for a vertical scanning LTP (VSLTP) was developed by Continental Optical Corporation under a Phase I SBIR program from NASA Marshall Space Flight Center. 19 . A standard LTP II 
system was modified to incorporate a vertical-scanning penta prism, and a precision rotation stage was added to provide the capability to produce a complete 3D map of the entire surface.

A schematic of the VSLTP prototype system is shown in Fig. 2. A telescope mandrel external surface and a shell interior surface are shown as they. would each be set up for testing, although only one would be tested at a time. The shell is shown standing with its axis vertical. A small penta-prism is scanned into the shell on a vertical slide. A series of axial scans is made at equally-spaced azimuthal angles, and then a set of azimuthal scans is made at selected axial height positions. The axial and azimuthal slope scans can then be combined to generate a slope angle map of the surface, which can then be integrated to produce a 3D height map of the surface.

A set of measurements with the VSLTP prototype was made on a NASA-supplied Wolter I telescope mandrel. Results of the measurements on the paraboloidal segment are shown in Figs. 3 and 4. The axial slope profiles are shown in Fig. 3. Each line consists of 36 data points measured at $1 \mathrm{~mm}$ intervals along the axial direction. The mandrel was rotated 10 degrees between each axial scan. Following completion of the axial scans, a set of azimuthal scans was made (not shown). The slope profiles were converted into height and the endpoints of each axial scan were fixed to the corresponding points on the appropriate azimuthal scans. The ideal paraboloidal surface was subtracted from the measured surface and the residual points are plotted in Fig. 4. The maximum peak-to-valley excursion in the residual surface is about $0.3 \mu \mathrm{m}$. The repeatability of the prototype measurements is estimated to be about 50 $\mathrm{nm}$ RMS in height and about $1 \mu \mathrm{rad}$ in slope for this set of measurements. Significant improvement in performance is expected in the next generation VSLTP which is being produced under a Phase II SBIR award from NASA MSFC to Continental Optical Corp.

\section{SUMMARY}

A number of measuring instruments and techniques are available for measuring the figure of $\mathrm{x}$-ray telescope optics. We have demonstrated that simple modifications to the standard LTP II configuration produce a more stable and repeatable scan and enable the uses of the LTP to be expanded to encompass in situ measurements and vertical scan measurements. The test requirements for current $x$-ray telescope fabrication projects want a slope error in the range of 0.5 to 1 arc second, but the PPLTP has the potential for measurement accuracy of 0.2 arc second ( $1 \mu \mathrm{rad})$ or less. A new 3-D VSLTP will be suitable for the testing of space $x$-ray optics.

\section{ACKNOWLEDGMENTS}

This research was sponsored in part by NASA Marshall Space Flight Center under SBIR contract number NAS8-40514 and in part by the U.S. Department of Energy: Contract No. DE-AC02-76CH00016.

\section{REFERENCES}

1. P. Gorenstein, "High throughput X-ray optics - An overview," Appl. Opt. 27, pp. 1433-1439, (1988)

2. B. Aschenbach, "Design, construction, and performance of the Rosat high-resolution X-ray mirror assembly," Appl. Opt. 27 (8), pp. 1404-1413, (1988)

3. M.R. Sims, M.A. Barstow, J.P. Pye, A. Wells, and R. Willingale, "XUV wide field camera for ROSAT," Qpt.Eng, 29 (6), pp. 649-657, (1990)

4. N.J. Westergaard, et al., "Status of the development of a thin-foil, high-throughput x-ray telescope for the Soviet Spectrum X-Gamma mission," in X-Ray/EUV Optics for Astronomy and Microscopy, Richard B. Hoover, ed., Proc. SPIE 1160, pp. 658.665 (1990)

5. K. Becker and E. Heynacher, "M400 - A coordinate measuring machine with 10 nm resolution," in Inprocess Optical Metrology for Precision Machining. Proc. SPIE, 802, pp. $209-216$ (1987)

6. A. Franks, "Nanometric surface metrology at the National Physical Laboratory," Nanotechnology 2, pp. 1118, (1991) 
7. H. Kunieda and P.J. Serlemitsos, "X-ray mirror assessment with optical light," Appl. Opt, 27, pp. 15441547. (1988)

8. K. von Bieren, "Interferometry of Wavefronts Reflected Off Conical Surfaces," Appl. Ont. 22, pp. 2109, (1983)

9. P. Z. Takacs and Shinan Qian, United States patent No.4884697 (5 Dec 1989)

10. P.Z. Takacs, S.-N. Qian, and J. Colber, "Design of a Long-Trace Surface Profiler," in Metrology - Figure and Finish, Bruce Truax, ed., Proc, SPIE 749, pp. 59-64 (1987).

11. P.Z. Takacs, S.-C.K. Feng, E.L. Church, S.-N. Qian, and W.-M. Liu, "Long trace profile measurements on cylindrical aspheres," in Advances in Fabrication and Metrology for Optics and Large Optics, Jones B. Arnold and Robert A. Parks, ed., Broc. SPIE 266, pp. 354-364 (1989)

12. S.C. Irick, W.R. McKinney, D.L.T. Lunt, and P.Z. Takacs, "Using a straightness reference in obtaining more accurate surface profiles", presented at Fourth International Conference on Synchrotron Radiation Instrumentation, 15-19 July 1991, Chester, United Kingdom, I.H. Munro and D.J. Thompson, ed., Rev. Sci. Insts.. Vol. 63, pp. 1436-1438, (1992), American Institute of Physics.

13. W. McKinney, S.C. Irick, and DJ. Lunt, "XUV synchrotron optical components for the Advanced Light Source: Summary of the requirements and the developmental program." in Optics for High-Brightness Synchrotron Radiation Beamlines, Proc SPIE 1740, pp. 154-160 (1992)

14. S.C. Irick, "Improved measurement accuracy in a long trace profiler: Compensation for laser pointing instability," Nuclear Instruments and Methods in Physics Research A347, pp. 226-230, (1994)

15. S.C. Irick, "Determining surface profile from sequential interference patterns from a long trace profiler," Rev. Sci. Instrum. 63 (1), pp. 1432-1435, (1992)

16. Shinan Qian, Giovanni Sostero and Werner Jark, "Operation experience with the long-trace-profiler LTP II and improvements", Sincrotrone Trieste Report, 1994

17. S.-N. Qian, W. Jark, and P.Z. Takacs, "The Penta-Prism LTP: A Long-Trace-Profiler with Stationary Optical Head and Moving Penta-Prism," Review of Scientific Instruments 66 (3), pp. 2562-2569, (1995)

18. S.-N. Qian, W. Jark, P.Z. Takacs, K.J. Randall, and W.-B. Yun, "An In-Situ Surface Profiler for High Heat Load Mirror Measurement," Opt. Eng. 34 (2), pp. 396-402, (1995)

19. H. Li, X. Li, M.W. Grindel, and P.Z. Takacs, "Measurement of X-ray Telescope Mirrors Using A Vertical Scanning Long Trace Profiler," Opt.Eng. 35 (2), pp. 330-338, (1996) 

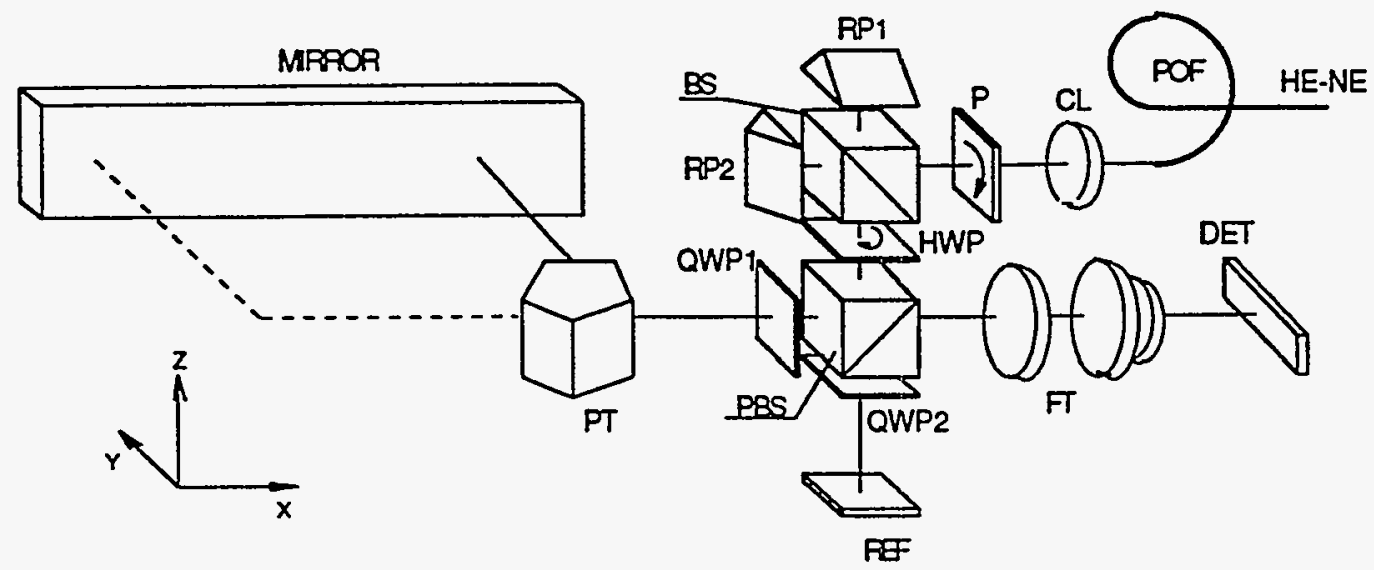

Fig. 1. Schematic of the Penta Prism LTP (PPLTP) configuration. The penta prism (PT) is the only moving part in this configuration. All other components of the optical head are mounted on a stationary platform.

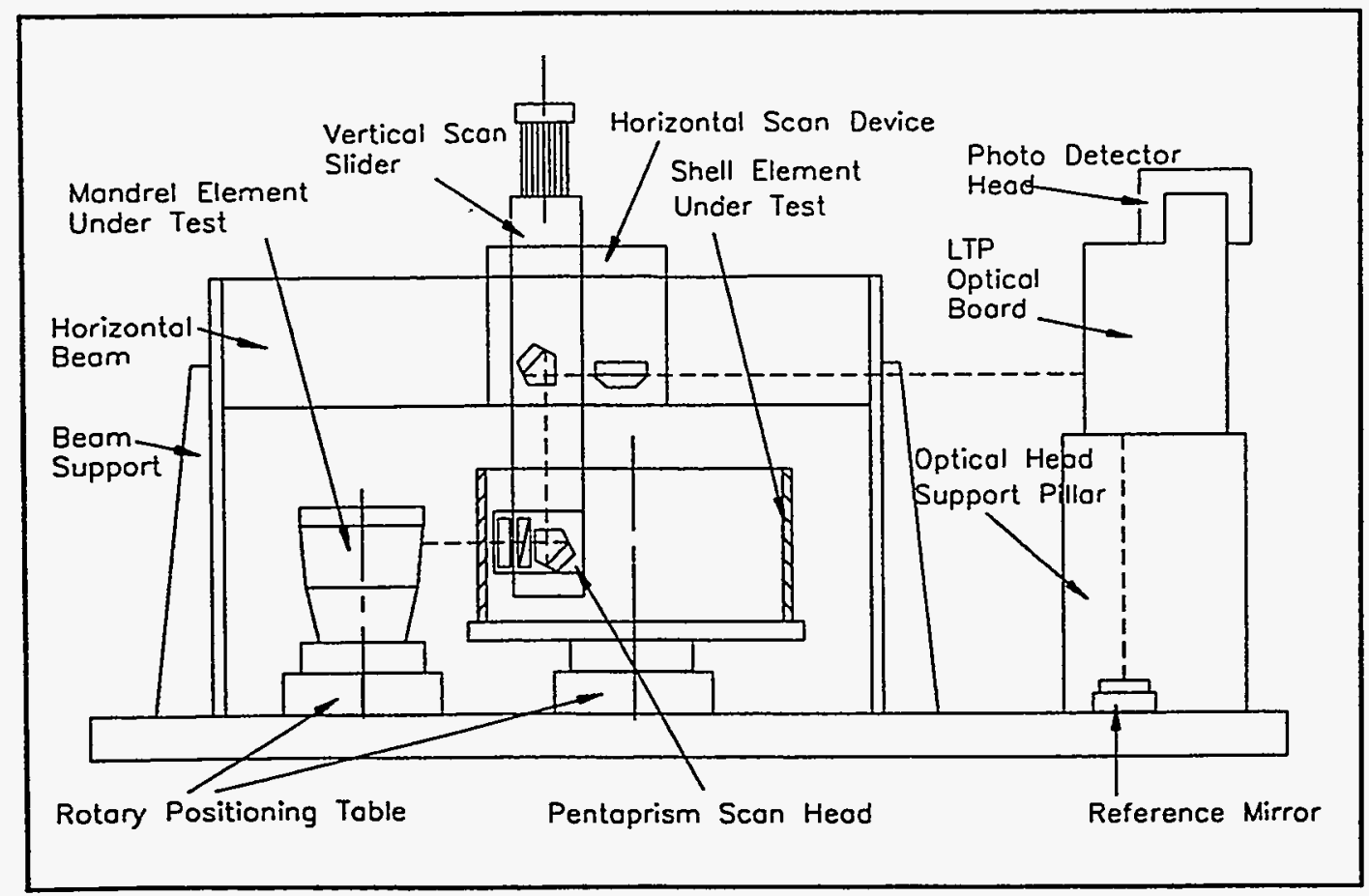

Fig. 2. Schematic of the Vertical Scan LTP (VSLTP) prototype instrument showing how measurements are made on telescope shells and mandrels mounted in a vertical configuration on a rotary stage. The optical head is mounted on a stationary platform off to the side. 


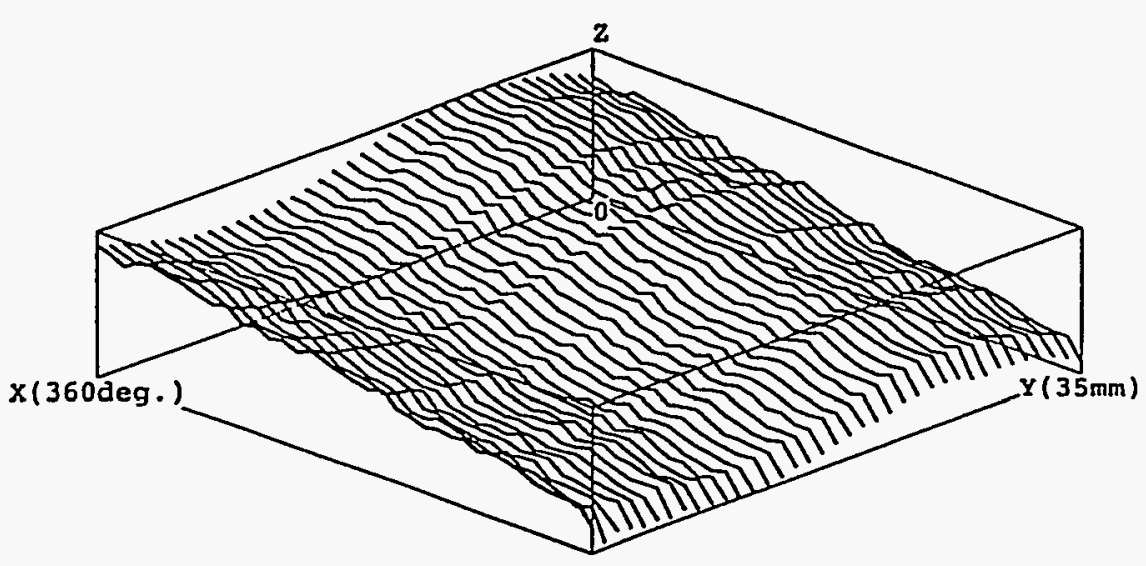

\begin{tabular}{|c|c|c|c|c|c|}
\hline File Name: & HLIYB,INE & Comments: & vertical w/o doy & m/lodeg & \\
\hline Data Type: & Slope & History: & I2S-RSE & & \\
\hline Frames (in X): & 37 & Frames (in $\mathbf{Y}$ ): & $\underline{36}$ & & \\
\hline Xmin: & $\underline{0,000000}$ & $X \max :$ & 360.000000 & X Unit: & degree \\
\hline Ymin: & 0,000000 & Ymax: & $\underline{35.000000}$ & Y Unit: & $\mathrm{mm}$ \\
\hline Zmin: & $5.791489 \mathrm{e}-003$ & Zmax: & $\underline{6,447094 \mathrm{e}-003}$ & Z Unit: & Slope (rad) \\
\hline
\end{tabular}

Fig. 3. Axial slope profiles on a Wolter I telescope mandrel made with the VSLTP prototype instrument. A total of 37 scans were made at $10 \mathrm{deg}$ increments in azimuth. Each scan consists of 35 points sampled at $1 \mathrm{~mm}$ intervals. The P-V slope change is about $0.65 \mathrm{mrad}$ over this data set and indicates the convex nature of the paraboloidal segment measured.

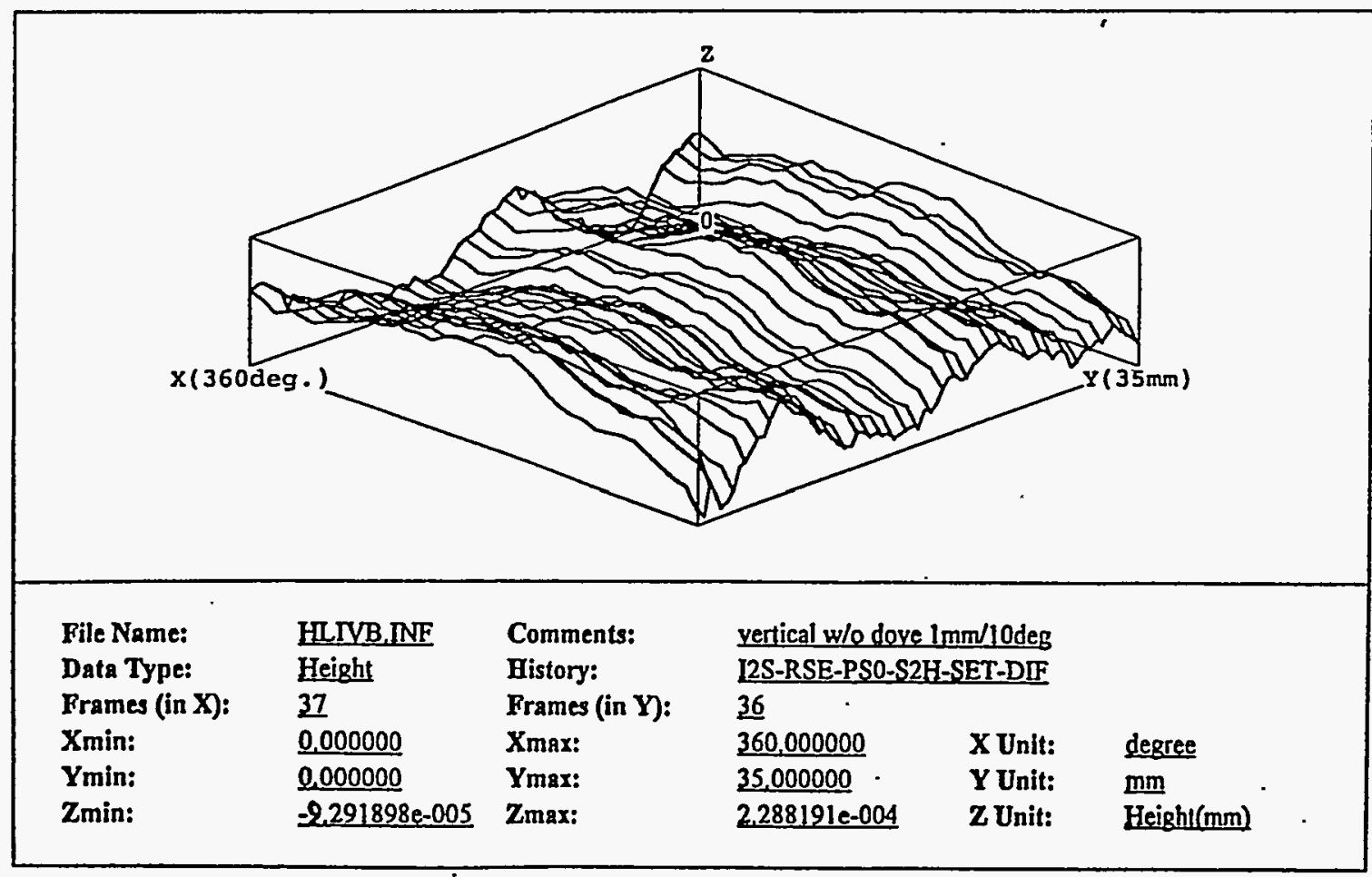

Fig. 4. Residual height of the mandrel surface after fixing the endpoints with azimuthal scans, converting to height, and subtracting the prescribed surface. Maximum P-V deviation is about 0.3 microns from the ideal paraboloidal surface. 